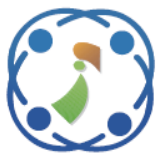

\title{
A Quasi-Mobile Sink Model to Optimize Deployment, Energy and Routing Cost for Scalable Static IoT Based Wireless Sensor Network
}

\author{
Jayavignesh Thyagarajan ${ }^{1 *}$ \\ Subashini Sundararajan ${ }^{1}$ \\ ${ }^{1}$ School of Electronics Engineering, Vellore Institute of Technology, Chennai Campus, India \\ * Corresponding author's Email: jayavignesh.t@ vit.ac.in
}

\begin{abstract}
Multi-hop based Wireless sensor network (WSN) has gained significant importance catering to large scale application demands and integrated with Internet of Things (IoT) in recent times. Conventional WSN faces hotspot problem, since nodes in the one-hop neighbourhood of static sink faces battery depletion rapidly for relaying other nodes' traffic. Mobile sink based approaches suffer from excessive control overhead in terms of topology updates, route computation and maintenance by sensor nodes. This work proposes a novel quasi-mobile sink query driven model to optimize cost factors such as deployment, energy and routing for large scale soft real-time applications. A corona based beacon-less flexible tree routing is adopted. In this model, sink moves in a predefined pattern covering the deployment zone but remains stationary for a configurable sojourn time during the query dissemination and data gathering phase. It is inferred from simulation results that significant energy savings is achieved over the hotspot region near the sink, with increase in traffic rate compared to static sink solution. High data delivery ratio and energy efficiency is achieved with increase in network size in comparison with existing sink mobility models. Empirical range test measurements of average Received Signal Strength Indicator (RSSI), Packet Success Rate (PSR) are also performed to determine the optimal transmission power level and ensure proper deployment for effective coverage and network connectivity.
\end{abstract}

Keywords: RSSI, WSN, Mobile sink, Deployment cost, Energy efficiency, Routing.

\section{Introduction}

With the recent advancement in network technologies and availability of miniaturized lowcost wireless sensor devices, the IoT technology has enabled to connect any object to the Internet. This facilitates machine-to-human and machine-tomachine communication in the physical world. WSN is an important component of the IoT and represents the key technology for the next generation Internet and computing devices [1].

Large Scale WSN applications demand the deployment of small battery powered sensor nodes populated in close proximity to the physical phenomenon in a dense manner. These applications are either periodic or event-driven and mostly pushpull driven protocol models [2]. The Sink disseminates the queries to the deployed sensor network and sensors which matches the interest queries generate many to one type of converge-cast traffic pattern towards sink. This creates hotspot region since sensors closer to sink are required to forward huge traffic for sensors farther from the sink.

Wireless multi-hop based sensor networks [3] imposes numerous challenges such as link instability (temporal and spatial variations caused due to random radio signal propagation), limited bandwidth, limited battery power, node's mobility pattern, speed variations and failure leading to dynamic topology changes. This necessitates the design of efficient multi-hop routing and routing metrics which impact the Quality of service (QoS) metrics directly for IoT Based Wireless Sensor Network Applications.

The significant contributions in this work are as follows.

1. A novel Quasi mobile sink's programmed movement pattern to reduce the deployment, 
energy and routing cost for scalable static WSN applications.

2. Query Dissemination by Quasi-mobile sink during sojourn time and data routing for matched-query interest source nodes in multihop manner. The forwarding parent node choice is based on RSSI routing metric and adopts a corona-level based tree routing mechanism. Simulation of the proposed work is carried out to show significant energy savings and high data delivery ratio. It is found suitable for soft realtime large scale static WSN applications.

3. Optimal transmission power level selection algorithm for homogeneous sensor nodes to operate in the region of deployment, supported by empirical measurements of performance metrics.

The rest of the paper is organized in the following sections. Section 2 presents the survey of existing works on deployment strategies, sink mobility models, routing and routing metrics. Section 3 presents the assumptions of the work, problem statement, proposed model and algorithms. Section 4 analyses the experimental and simulation results. Section 5 presents the conclusion and future work to be carried out.

\section{Related work}

\subsection{Sensor network deployment related works}

Numerous sensor network deployment strategies [4 - 7] are proposed in the literature to maximize the key performance metrics of WSN. Cheng et al. [4] proposed general network lifetime and deployment cost models to evaluate the various WSN deployment strategies and emphasize that a deployment strategy is good only if able to achieve both energy savings and energy balance. The maximum achievable network lifetime decreases, if energy imbalance occurs and cannot be suited for large scale WSN applications. This work also took into consideration that transmission power control mechanism strategy alone is not sufficient to solve both energy savings and energy imbalance problem.

Non-uniform energy assignment for nodes in hot spot region to achieve both energy efficiency and energy-balance is not a practical solution.

[5] proposed engineered gaussian corona based deployment strategy to mitigate hot-spot problem using arithmetic and geometric proportions such that the density of sensor nodes in inner corona i.e. sink's neighbourhood is higher compared to outer level coronas.
Though several smart deployment strategies are proposed in literature [5 - 7] and claims better network lifetime and energy savings, the cost involved in implementing these strategies are mainly ignored. The required number of sensor nodes to achieve full sensing coverage and network connectivity is extensively analysed with several deployment patterns [7].

Multiple sinks based strategies could improve network lifetime as more sinks may lead to only few multi-hops [8]. This strategy could make it suitable for time-sensitive applications, but can increase the overall deployment cost.

The primary focus of several deployment strategies based works has only been to meet the Quality of Service (QoS) metrics demands but forgets to evaluate the cost factor incurred for a practical WSN deployment.

\subsection{Mobile sink based dynamic routing works}

A unified framework is built in [9] to maximize the network lifetime in WSN by jointly considering the sink mobility and routing involving single mobile sink. It is proved that moving the sink is always better than keeping them static to achieve energy savings in network. [8] introduces mobile data collectors and determines path to cover the full network in order to collect data from sensors in single hop. This work exploits the trade-off between latency and energy consumption by considering multiple mobile sinks. Pure mobile sink based trajectory solutions such as proposed in [10] suffers from longer traversing time as data can be delivered to the sink only when they come closer to the boundary and almost have no multi-hopping.

Abdul et al. [11] presents a comprehensive survey and taxonomy of various data dissemination and data collection schemes that exploits mobility of sink. Controlled, random, fixed path mobility patterns are analysed for its suitability to time-sensitive and delay tolerant applications.

Controlled Mobility schemes is based on controlling or guiding the sink movement based on the parameter of interest or objective function or observable events in the network [12, 18]. The communication required to gather information regarding the parameter of interest add to the extra overhead. Fixed path schemes are programmed to follow a fixed path and is not influenced by the network behaviour at run-time.

Multi-hop routing protocols are either proactive, reactive, query driven, hybrid, cluster-driven or geographical based to combat energy-delay trade-off factors [12 - 14]. The energy cost incurred due to 
frequent topology updates w.r.to sensor node mobility, sink's mobility and re-transmissions due to poor link quality affects the QoS metrics of WSN. In [15], a corona based tree routing mechanism is adopted, where the sink mobility is random. The sensor nodes transmits periodic beacons for neighbourhood management which incurs more energy in-terms of topological updates and extra signalling overhead for routing metrics computation.

Majid et al. [16] focuses on a careful selection of duty cycle value and mobility radius of mobile sink to minimize the maximum energy dissipation and average energy dissipation of all nodes in network.

Lei Shi et al. [17] adopts a novel data driven routing approach with mobile sinks which relies on overhearing data transmission/beacons for forming routes or does random-walk to reach sink. This suffers from energy wastage due to interference, hidden terminal issues, re-transmissions and unbounded end to end delay.

Abdala et al. [18] proposes distance based controlled sink mobility (DBCSM) by moving sink towards farthest nodes which have high remaining energy and lower load. This work suffers data delivery loss due to unstable links and connectivity with mobile sink.

\subsection{Routing metric design based works}

Minimize the routing cost without losing connectivity in the dynamic topology is the design goal of routing metrics. Radio links are unreliable in low power WSN and metrics to estimate link quality estimation is necessary to mitigate the packet loss. Numerous link quality estimators [19] which are asymmetric aware and interference aware were studied such as LQI (Link Quality Indicator), RSSI (Received Signal Strength Indicator), SINR (Signal to Interference plus Noise Ratio), ETX (Expected Transmission Count) etc. The probe messages and the extra overhead involved in estimating link quality adds significantly to the energy consumption per node.

Hardware based link quality estimator such as RSSI represents the received signal strength observed during packet reception at the receiver's antenna. It can be directly read from the radio transceiver and does not require any additional computation. RSSI $[20,21]$ is one of the important metric used by upper layers for distance estimation for next hop forwarding node choice to sink, link quality estimation, and localization scheme such as trilateration.

\section{Problem statement}

The sensor network field is considered with the following assumptions.

1. Sensor nodes are static, uniformly deployed with minimum separation distance between nodes computed based on range test inferences to ensure network connectivity and area coverage without holes.

2. Sensor nodes are resource constrained in terms of limited battery power, memory, processing capability and bandwidth.

3. Sink is assumed to be free of such constraints except for bandwidth and programmed to move in a preconfigured pattern.

4. The query dissemination interval is fixed and periodic by the sink depending on soft-real time applications' requirement. Dissemination interval is set once every sojourn time interval depending on the application demands on hourly, daily or monthly basis.

5. The deployment environment is considered to be two-dimensional squared area.

The problem consists of 4 parts

1. Optimal transmission power level selection algorithm for configuring all the homogeneous sensor nodes before deploying in the environment.

2. Quasi mobile sink's programmed movement pattern.

3. Query Dissemination by Quasi-mobile sink during sojourn time alongside route tree construction.

4. Data routing for matched-query interest source nodes in multi-hop manner. The forwarding parent node choice is based on RSSI metric recorded during the route tree construction.

\subsection{Optimal transmission power level selection algorithm}

The part 1 of the work focuses upon the empirical evaluation using Xbee IEEE 802.15.4 radio transceiver [23] for wireless communication. This transceiver can set the transmission power level $\left(\mathrm{P}_{\mathrm{t}}\right)$ in order to minimize energy consumption and increase the radio range. To calculate the optimal transmission power level setting the sensor nodes should operate, range tests were performed in different environment depending on the application deployment requirement and transmission power level was selected according to the Algorithm 1

\section{Algorithm 1: Optimal Transmission Power Selection Algorithm \\ 1: function RANGETEST(RSSI, PSR, d)}


2: // RSSI - Received Signal Strength in dBm

3: // PSR - Packet Success Rate in \%

4: // $\mathrm{d}-$ one hop distance separation in meters

5: Initialize $\mathrm{d}=0$, numOfPktsRecvd $=0$;

6: $\mathrm{d}_{\max }=0 ; \quad / /$ Maximum effective one-hop communication range

7: numOfTxPkts=100;

8: numOfPktsLostErrored $=0$;

9: // minimum separation distance threshold value

10: $\mathrm{d}_{\text {thresh }}=$ constant

11: for $\mathrm{Pt}$ in $(-6 \mathrm{dBm},-4 \mathrm{dBm}, 0 \mathrm{dBm},+3 \mathrm{dBm}$, $+7 \mathrm{dBm}) \mathrm{do}$

12: $\quad$ for $\mathrm{d} \leftarrow \mathrm{d}+10$ meters do

13: for $\mathrm{i}=1$ to numOfTxPkts do

14: $\quad$ // RSSI $\quad$ current represents recorded RSSI of the currently received packet

15: $\quad$ RSSI $_{\text {sum }}=\mathrm{RSSI}_{\text {sum }}+\mathrm{RSSI}_{\text {current }}$

16: if (Packet received without error) then

17: $\quad$ numOfPktsRecvd++;

18: $\quad$ else

19:

20:

21:

22:

numOfPktsLostErrored++;

end if

end for

if (numOfPktsLostErrored $\geq$ $\mathrm{p} \times$ numOfTxPkts) then

23: $\quad$ // where $0.5 \leq \mathbf{p} \leq \mathbf{1}$

24: $\quad$ if $\left(\mathrm{d}>\mathrm{d}_{\max }\right)$ then $\mathrm{d}_{\max } \leftarrow \mathrm{d}$

25: $\quad$ end if

26: break; // Perform the next iteration/test

27: $\quad$ end if

28: $\quad$ end for

29: $\quad \operatorname{PSR}[\mathrm{d}]=\frac{\text { numOfPktsRecvd }}{\text { numOfTxPkts }}$

30: $\quad \operatorname{RSSI}_{\mathrm{avg}}[\mathrm{d}]=\frac{\mathrm{RSSI}_{\text {sum }}}{\text { numOfPktsecvd }}$

31: end for

32: Select optimal $\mathrm{P}_{\mathrm{t}}$ with min $\mathrm{d}_{\max }$ among $\forall \mathrm{P}_{\mathrm{t}}$ values such that $\min \mathrm{d}_{\max } \geq \mathrm{d}_{\text {thresh }}$

\section{3: end function}

The range test is performed by varying the onehop separation distance between the nodes and the average RSSI and PSR for transmission of 100 packet samples are computed. The range test is repeated for the different transmission power levels and the optimal power level for sensor nodes to operate on is selected based on minimum $\mathrm{d}_{\max }$ among all $\mathrm{P}_{\mathrm{t}}$ values, such that minimum $\mathrm{d}_{\max } \geq \mathrm{d}_{\text {thresh }}$ (minimum separation distance between the sensor nodes) to optimize the overall deployment cost. This power level selection decision is influenced depending on nature of deployed environment (Indoor / Outdoor Line of Sight (LOS)/ Non-Line of Sight (NLOS), dense foliage etc.).
Let the maximum effective one hop range (with acceptable Packet Success Rate (PSR) and Average RSSI > Receiver Sensitivity) using optimal transmission power level $\mathrm{P}_{\text {opt }}$ be ' $\mathrm{d}$ ' meters. To ensure network connectivity and optimize deployment cost, a minimum of two nodes are required to cover a square field of side $\sqrt{2} \mathrm{~d}$ meters, where diagonal ' $\mathrm{d}$ ' meters represents the maximum effective transmission range. Hence to ensure network connectivity, a minimum of 2 nodes are required for a deployment area of $\frac{d^{2}}{2}$ meters ${ }^{2}$.

\subsection{Proposed quasi-mobile sink model}

A cost effective WSN model is to adopt a single sink instead of multiple sinks. However, to avoid the hotspot around static sink, quasi-mobile sink model is proposed. In this proposed model, sink is stationary during the query dissemination and data collection phase. After this sojourn period, sink moves around the periphery of the deployment zone. The movement pattern around the square deployment area and possible combinatorics of movement patterns are shown in Figs. 1 and 2. The average mobility speed of sink $(v)$ is determined based on the data collection frequency (' $T$ ' time-units), peripheral distance to move from one extreme end of the area to the other end ('D' meters). It should be significantly greater than $\frac{D}{T}$.

The sink is quasi-mobile, since it moves to the extreme ends of the deployment zone $(0,0),\left(x_{\max }, 0\right)$, $\left(\mathrm{x}_{\max }, \mathrm{y}_{\max }\right),\left(0, \mathrm{y}_{\max }\right)$ for the first four rounds, where it remains stationary during the data collection. $\left(\mathrm{x}_{\max }, \mathrm{y}_{\max }\right)$ represents maximum dimensions of 2D deployment area. In the final fifth round, sink is

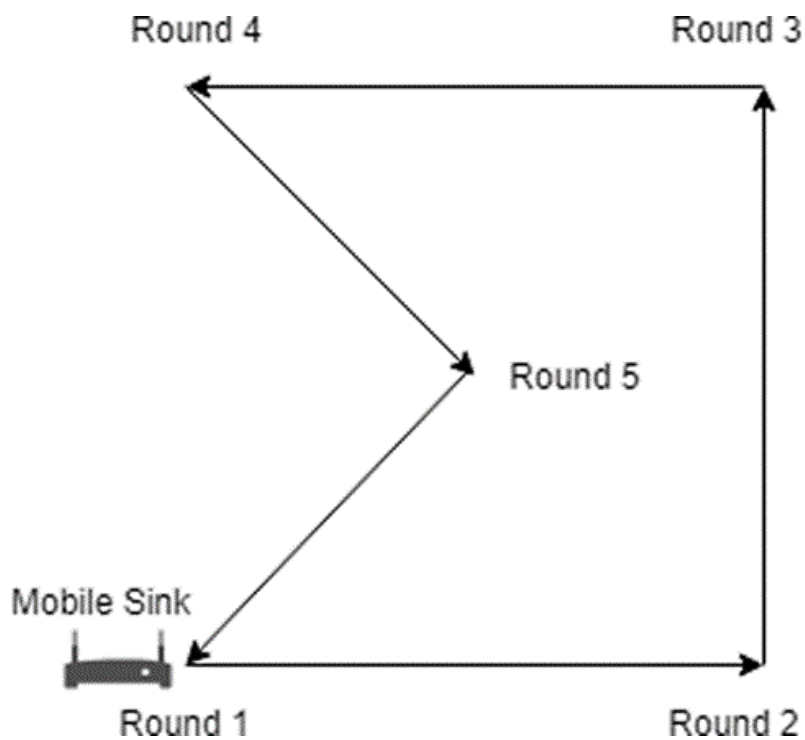

Figure. 1 Quasi mobile sink movement pattern and sojourn rounds 

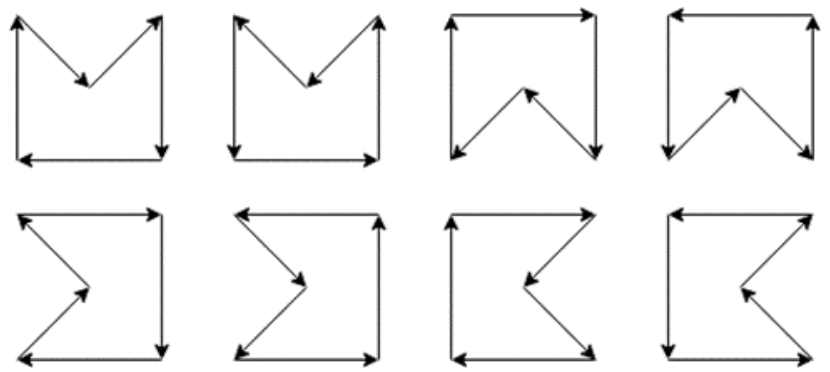

Figure. 2 Combinatorics of sink's pre-configured mobility pattern

made to move to the center of deployment zone $\left(\frac{x_{\max }}{2}, \frac{y_{\max }}{2}\right)$ for data collection. The limited number of sojourn rounds is decided to balance the topological query updates overhead and energy consumption. These rounds are repeated in cyclic pattern till the lifetime of application.

The rounds (sojourn time) and movement patterns are programmed and made configurable either in terms of hours, days or months depending on the periodicity of data collection in the deployed zone. The algorithm for Quasi Mobile Sink node movement pattern in presented in Algorithm 2.

\section{Algorithm 2: Quasi Mobile Sink Movement}

1: function QuasiMobileMovement $(\mathbf{x}, \mathbf{y}, \mathbf{t}, \mathbf{v})$

2: // where $(\mathrm{x}, \mathrm{y})$ - Sink current 2D coordinates

3: $/ / \mathrm{t}-$ idle time in units (minutes/hrs/day) to remain static

4: $/ / \mathrm{v}-$ maximum speed in meters/sec

5: // $\left(\mathrm{x}_{\max }, \mathrm{y}_{\max }\right)-$ maximum dimensions of $2 \mathrm{D}$ deployment area

6: Initialize $\mathrm{x}=0, \mathrm{y}=0, \mathrm{t}=\mathrm{constant}$;

7: numOfRounds $=5$;

8: for $\mathrm{i}=1$ to numOfRounds do

9: $\quad$ if $(x==0) \& \&(y==0)$ then

10: $\quad \mathrm{x}=\mathrm{x}_{\max }$

11: Move sink at ' $\mathrm{v}$ ' speed units to reach $\left(\mathrm{x}_{\max }, \mathrm{y}\right)$

12: $\quad$ Call Query Dissemination Route Tree Construction procedure

13: $\quad$ idle $(\mathrm{t}) / /$ Sink remains in this position for time ' $t$ ' units

14: end if

15: $\quad$ if $\left(x==x_{\max }\right) \& \&(y==0)$ then

16: $\quad \mathrm{y}=\mathrm{y}_{\max }$

17: Move sink at ' $v$ ' speed units to reach $\left(\mathrm{x}, \mathrm{y}_{\max }\right)$

18: $\quad$ Call Query Dissemination Route Tree Construction procedure

19: $\quad$ idle(t) // Sink remains in this position end if for time ' $t$ ' units

21: $\quad$ if $\left(\mathrm{x}==\mathrm{x}_{\max }\right) \& \&\left(\mathrm{y}==\mathrm{y}_{\max }\right)$ then
22:

23:

24:

25:

26:

27:

28:

29:

30: $\quad$ Move sink at ' $\mathrm{v}$ ' speed units to reach $\left(\frac{x_{\max }}{2}, \frac{y_{\max }}{2}\right)$

31: $\quad$ Call Query Dissemination Route Tree Construction procedure

32: $\quad$ idle(t) // Sink remains in this position

33: $\quad$ end if for time ' $t$ ' units

34: end for

35: end function

\subsection{Query dissemination by quasi mobile sink and routing by sensor nodes $(\mathrm{SN})$}

Quasi mobile sink broadcast query packet containing the interest query, Corona Level Identifier (CLID) during the sojourn time to one-hop neighbours, which they re-broadcast to next hop neighbours.

The sink broadcasts the CLID valued 0 and the next hop neighbours increments the level by 1 and rebroadcasts. This helps every sensor node to form a

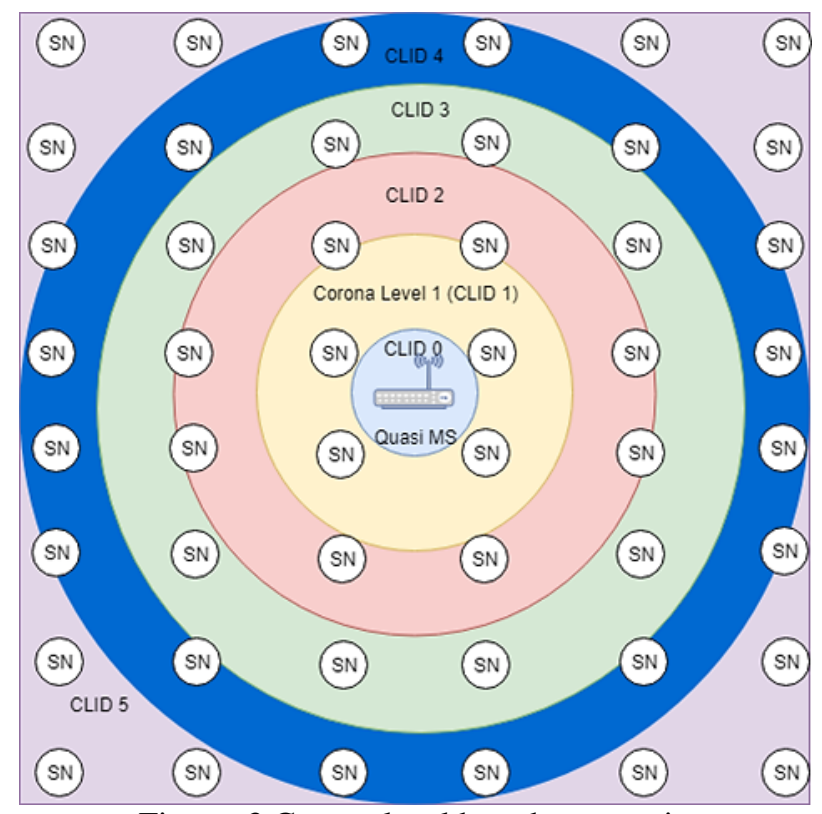

Figure. 3 Corona level based tree routing 


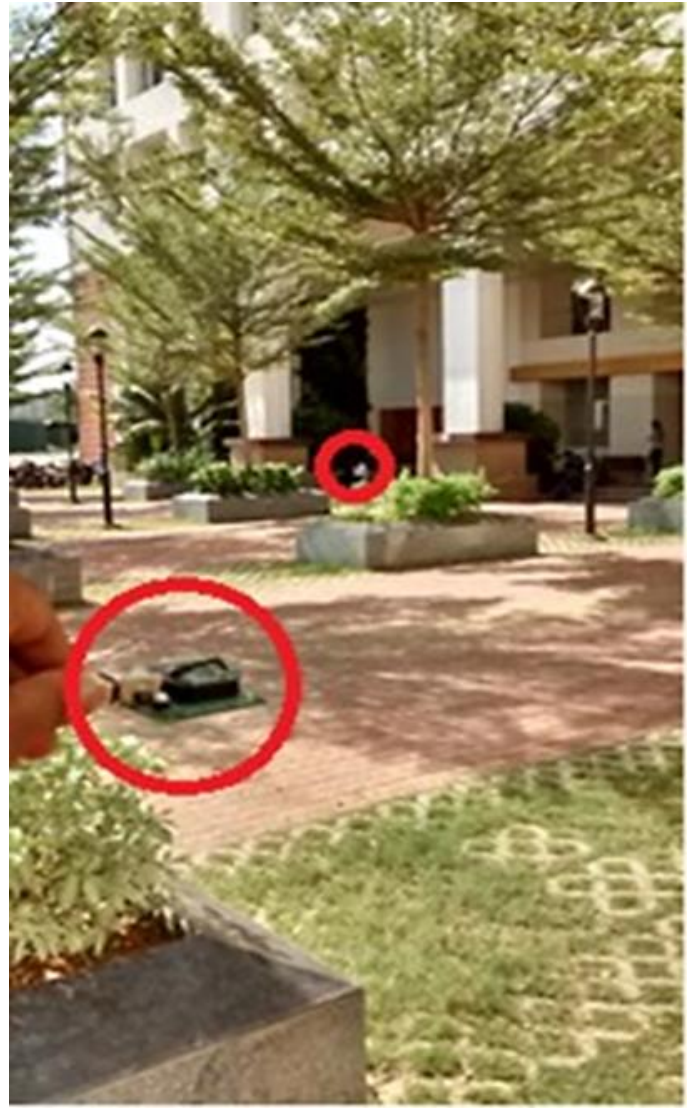

Figure. 4 Range test in outdoor environment

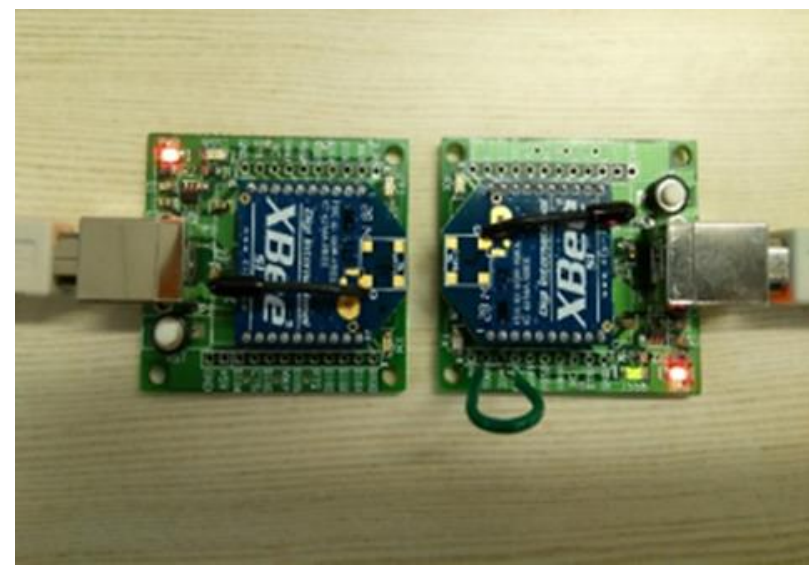

Figure. $5 \mathrm{RSSI}_{\max }$ recording

neighbour table and determine the lower level nodes with their RSSI recorded while receiving the query packet. Upon interest query match by SN, they could choose parent node in lower level from neighbour table which has recorded a better RSSI and send their observed data. The routing process repeats till it reaches the sink at CLID 0.

Fig. 3 shows the round 5 where the quasi mobile sink is in middle of the zone and corona is defined as a concentric radio coverage w.r.to the sink.

The IEEE 802.15.4 MAC layer acknowledgement will ensure hop by hop delivery and energy wastage via re-transmission is avoided by choosing next hop node with better link quality. The corona level based routing is a flexible tree based routing, since it can choose parent node in same level to forward, if it fails to find parent node in lower level. The corona based relative level / distance from sink node is a better alternative to GPS based geographical routing schemes to reduce signalling overhead for mobile based WSN.

\section{Results and discussion}

\subsection{Pre-deployment range test experimentation}

An experimental study was conducted to evaluate and analyse the effective communication range, the choice of transmission power level (' $\mathrm{P}_{\mathrm{t}}$ ') for the homogeneous sensor nodes to operate in the region of deployment. The packet error rate variability with respect to the change in $\mathrm{P}_{\mathrm{t}}$, separation distance (' $\mathrm{d}$ ' meters), nature of environment (Indoor and outdoor with LOS and N-LOS characteristics) were observed. The RSSI window variation was also recorded.

\subsubsection{Experimental setup}

1. Two X-bee 802.15.4 radios were assigned the same PAN Identifier to form the network.

2. Packet Success Rate and RSSI parameters were recorded and monitored at the receiving end.

3. Packet Success Rate is a probabilistic event and hence recorded an average for 100 packet samples with transmit interval 500ms.

4. Range test experiments was repeated for different $P_{t}$ levels $-10 \mathrm{dBm},-6 \mathrm{dBm},-4 \mathrm{dBm},-2 \mathrm{dBm}$ and $0 \mathrm{dBm}$. For each $\mathrm{P}_{\mathrm{t}}$ level, measurements were taken for each value of distance separation and incremented by 10 meters for every iteration.

5. The $\mathrm{RSSI}_{\max }$ or reference RSSI result was obtained from range test when two modules were kept at the least possible distance separation. The RSSI $_{\max }$ recorded for different $\mathrm{P}_{\mathrm{t}}$ was ranging from $-31 \mathrm{dBm}$ to $-24 \mathrm{dBm}$.

6. For every $\mathrm{P}_{t}$ value, there is a specific range beyond which $\mathrm{X}$-bee radio falls out of range. Thus, no RSSI and success rate are observed giving the actual communication range for that $\mathrm{P}_{\mathrm{t}}$.

7. A sample range test performed in Outdoor environment and RSSI ${ }_{\max }$ recording is shown in Figs. 4 and 5.

8. The results recorded for $10 \mathrm{dBm}$ (lowest Pt) and $-4 \mathrm{dBm}$ (optimal choice) in different environment are only shown in Figs. 6-9 for the clarity of discussion. 


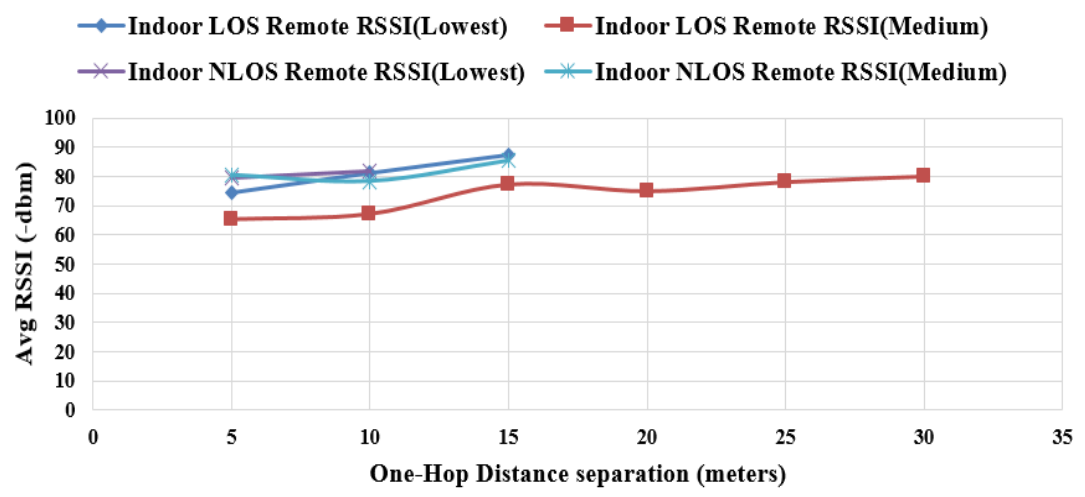

Figure.6 Average RSSI versus one-hop distance separation in indoor LOS and N-LOS environments for lowest and optimal Pt levels

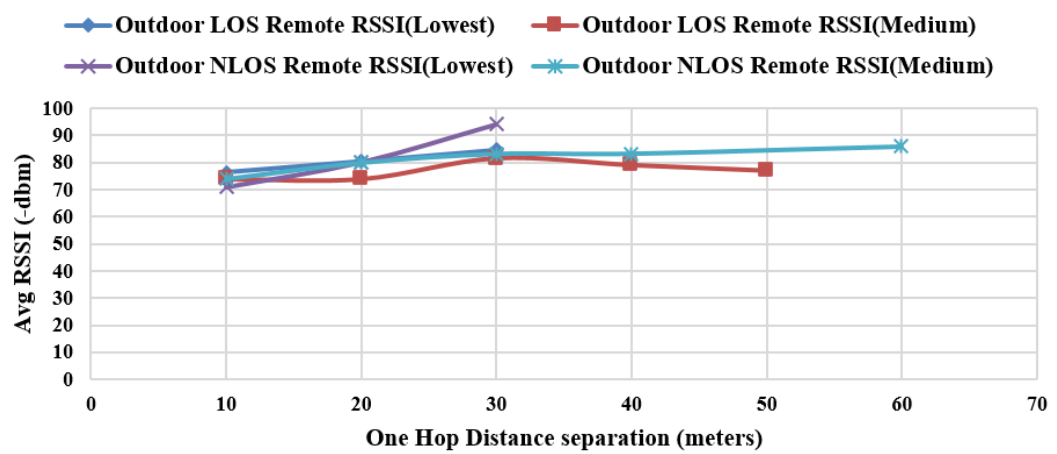

Figure.7 Average RSSI versus one-hop distance separation in outdoor LOS and N-LOS environments for lowest and optimal Pt levels

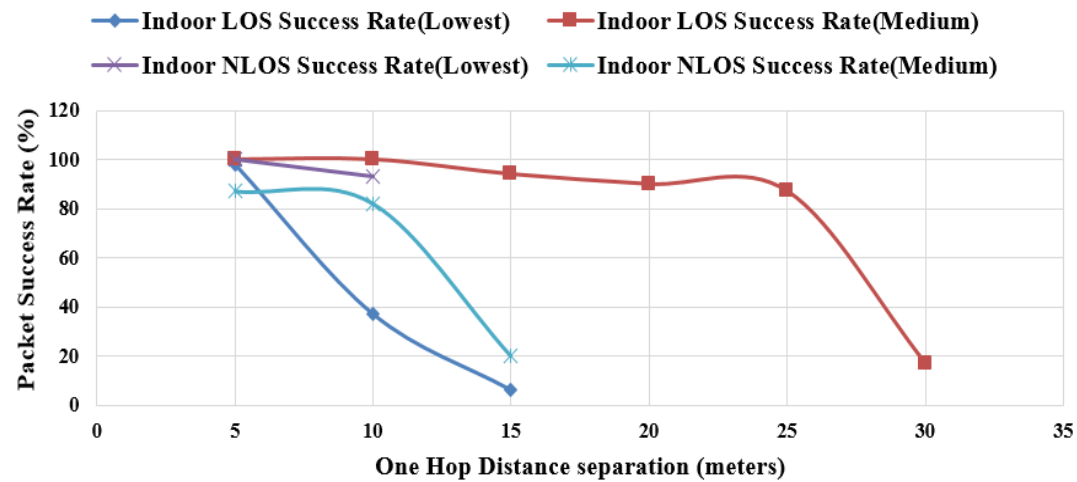

Figure. 8 Average PSR versus one-hop distance separation in indoor LOS and N-LOS environments for lowest and optimal Pt levels

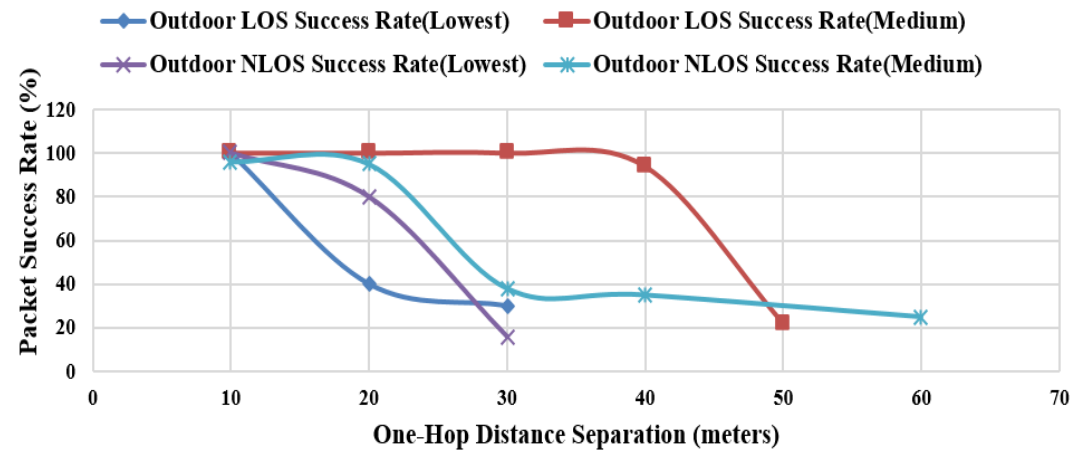

Figure.9 Average PSR versus one-hop distance separation in outdoor LOS and N-LOS environments for lowest and optimal Pt levels 
It is inferred from Figs. 6 and 7 that higher the transmit power level, the better the average RSSI and hence better link quality. But in order to design an energy efficient WSN, the nodes cannot be configured to run at higher power levels as it results in quick draining of batteries. Therefore, an optimal power level was selected in order to prolong battery life for a uniformly deployed static WSN.

It is inferred from Fig. 8, Fig. 9 that average PSR decreases with increase in distance separation and next hop node selection for routing should be chosen in such a way it maximizes the distance progress, minimizes the hop count and also does not affect the end to end packet delivery ratio.

Next Hop consideration for outdoor N-LOS can be done within 30 meters when operated at medium $\mathrm{P}_{\mathrm{t}}$ to minimize the hop count and within 40 meters for outdoor LOS environment at medium $\mathrm{P}_{\mathrm{t}}$. Similarly, the next hop consideration for Indoor LOS can be done between 10-15 meters using medium $\mathrm{P}_{t}$ and within 10 meters for Indoor N-LOS using medium $\mathrm{P}_{\mathrm{t}}$.

\subsection{Quasi-sink model - simulation \& analysis of multi-hop routing}

The sensor nodes are deployed in regular manner and spaced based on optimal transmission power level configuration and range testing results. The nature of environment is configured using Two ray ground radio propagation model using NS2 simulator with IEEE 802.15.4 MAC/PHY layers support. Table 1 shows the simulation parameters configured to all sensor nodes in NS2 operating in $2.4 \mathrm{GHz}$ unlicensed band.

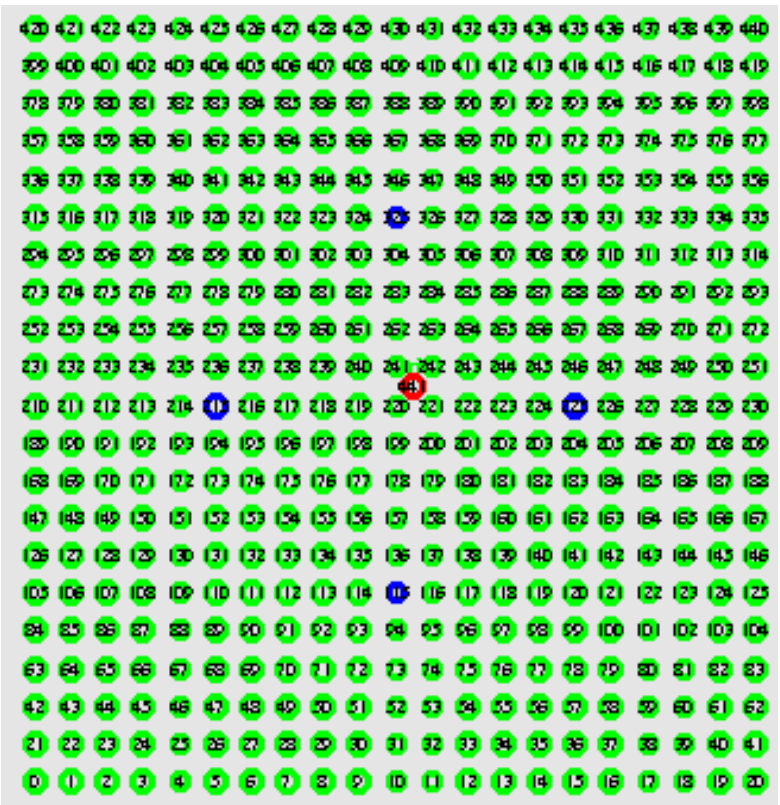

Figure. 10 Round 5 - Sink at center

\begin{tabular}{|l|l|}
\hline Operation mode & $\begin{array}{l}\text { Non-Beacon } \\
\text { (Unslotted) }\end{array}$ \\
\hline $\begin{array}{l}\text { Radio Propagation } \\
\text { Model }\end{array}$ & $\begin{array}{l}\text { Two Ray Ground } \\
\text { Reflection }\end{array}$ \\
\hline Transmit Power $\mathbf{P}_{\mathbf{t}}$ & $1 \mathrm{~mW}$ \\
\hline $\begin{array}{l}\text { Height of Tx/Rx Omni } \\
\text { Antenna }\end{array}$ & $1.5 \mathrm{~m}$ \\
\hline $\begin{array}{l}\text { Carrier Sense } \\
\text { Threshold }\end{array}$ & $4.39762 \mathrm{e}-10(15 \mathrm{~m})$ \\
\hline $\begin{array}{l}\text { Receiver Sense } \\
\text { Threshold }\end{array}$ & $4.39762 \mathrm{e}-10(15 \mathrm{~m})$ \\
\hline $\begin{array}{l}\text { Frequency of } \\
\text { Operation }\end{array}$ & $2.4 \mathrm{GHz}$ \\
\hline Initial Energy & $3.6 \mathrm{~J}$ \\
\hline TxPower/RxPower & $0.02955 / 0.0255 \mathrm{~W}[23]$ \\
\hline $\begin{array}{l}\text { Transport layer / } \\
\text { Traffic Type }\end{array}$ & UDP / CBR \\
\hline Packet Size & 70 bytes \\
\hline Packet Rate & 1,2 \& 5 packets/sec \\
\hline Simulation time & $250 \mathrm{sec}$ \\
\hline
\end{tabular}

Table 1. Simulation parameters in NS2

The sample simulation deployment pattern is shown in Fig. 10, where the sink marked as red and query matched source nodes are marked as blue.

\subsubsection{Simulation results and analysis with increase in} traffic rate

The network size was fixed to 121 nodes and the traffic rate with respect to fixed number of querymatched source nodes was increased to record the results.

Fig. 11 infers that the total energy consumption of network increases as the packet rate increases.

However, the cumulative energy consumption using quasi-mobile sink (QM Sink) approach is comparatively lesser than the static sink solution. It is observed around 20-40\% energy savings is achieved with increase in packet rate up to 5 packets /second per node in the proposed quasi-mobile sink model. Similarly, the average energy consumed in the network is also shown in Fig. 12.

Fig. 13 infers that about 5-6.5\% increase in energy savings with increase in packet rate up to 5 packets/second per node is achieved among the hotspot region near the sink in the quasi mobile sink model compared to static sink model. This approach minimizes the over-dissipation of battery power in hot spot region and balances the load among the nodes deployed through quasi mobile sink solution. 


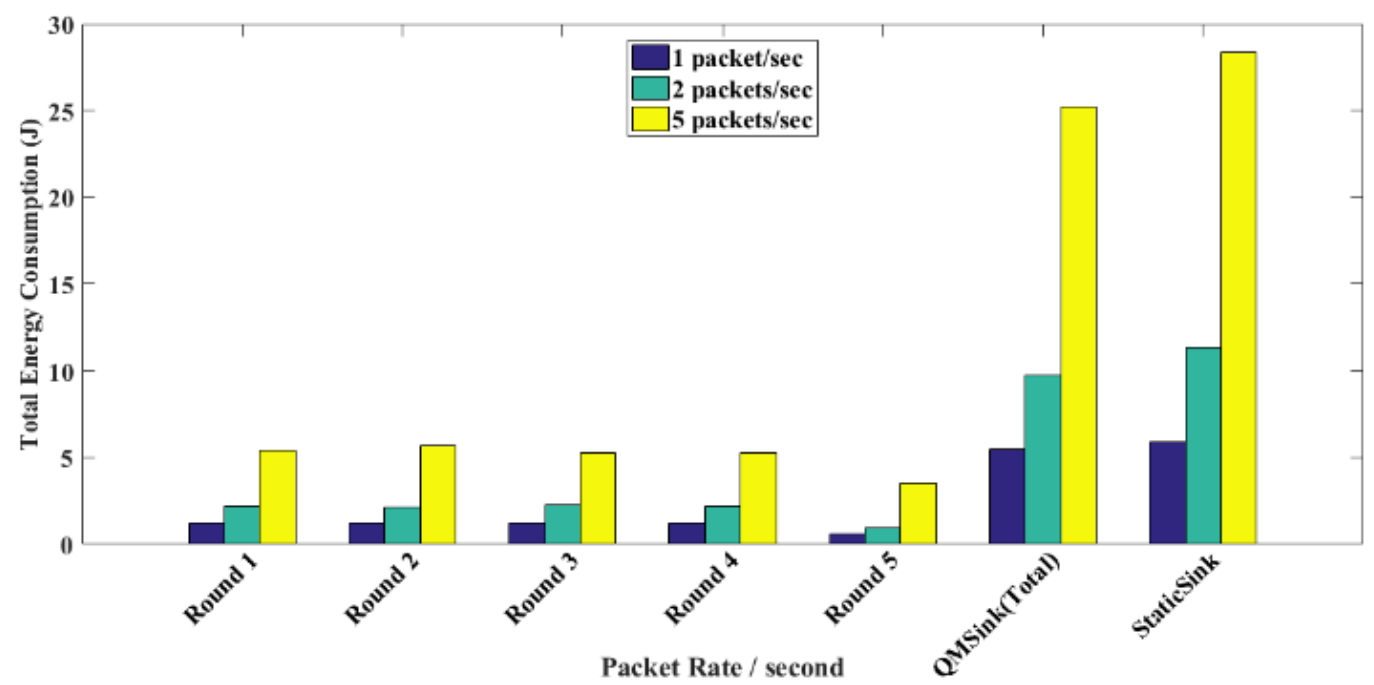

Figure. 11 Total energy consumption in WSN $(\mathrm{J})$ vs packet rate $(\mathrm{p} / \mathrm{sec})$

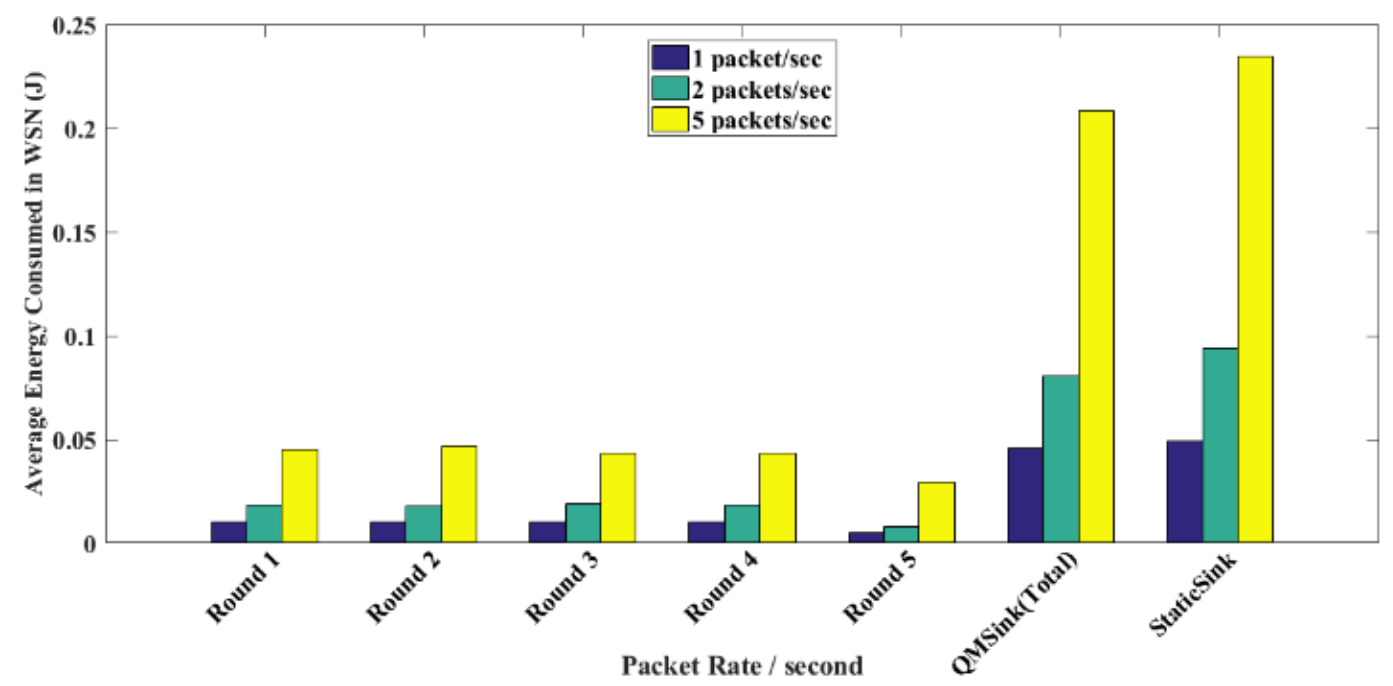

Figure. 12 Average energy consumed in WSN (J) vs packet rate (p/sec)

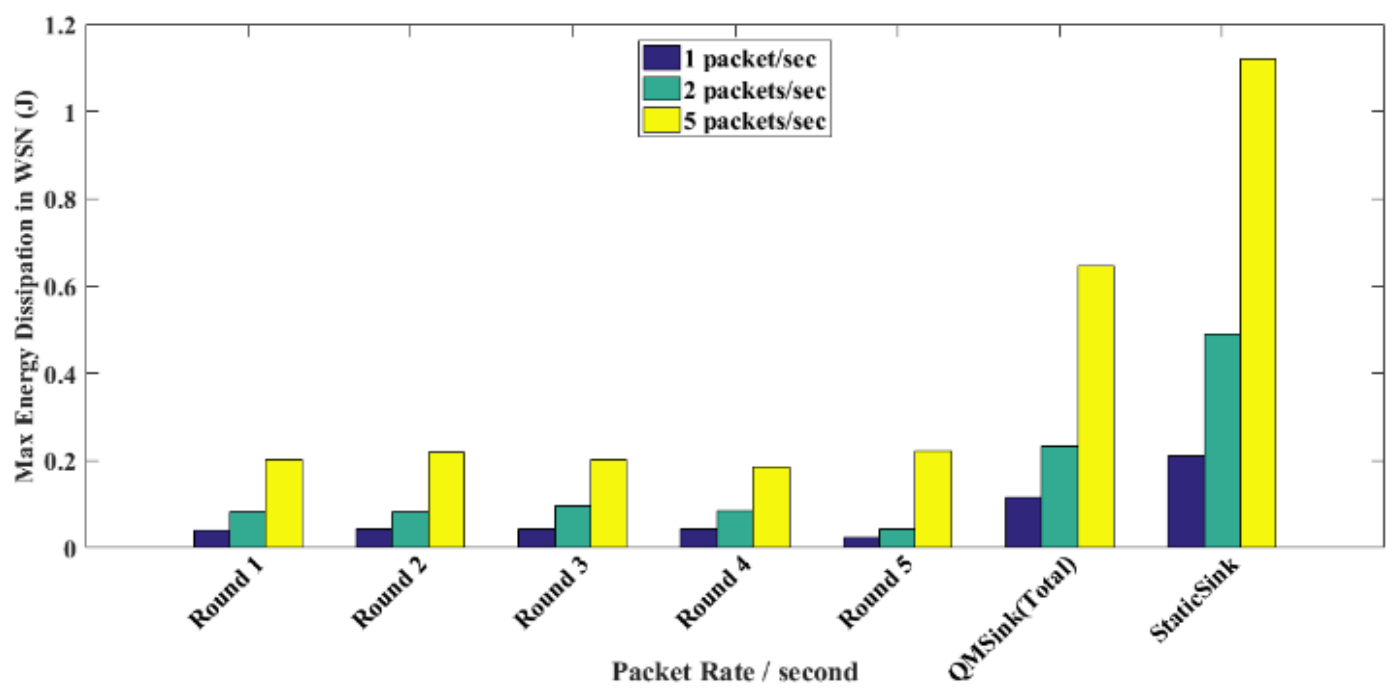

Figure.13 Hot spot node - Max energy dissipation in WSN (Joules) vs packet rate (p/sec) 


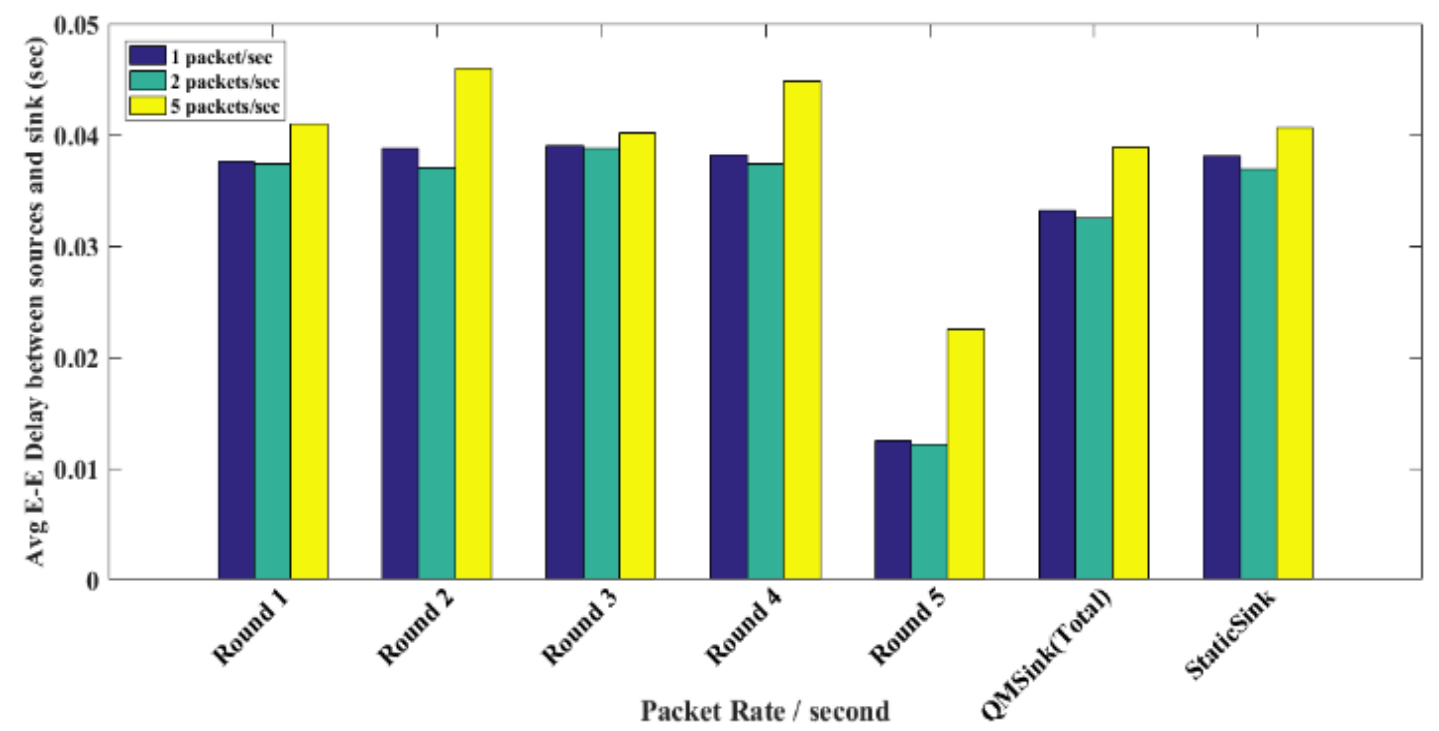

Figure. 14 Average end to end delay between sources \& sink (seconds) vs packet rate (p/sec)

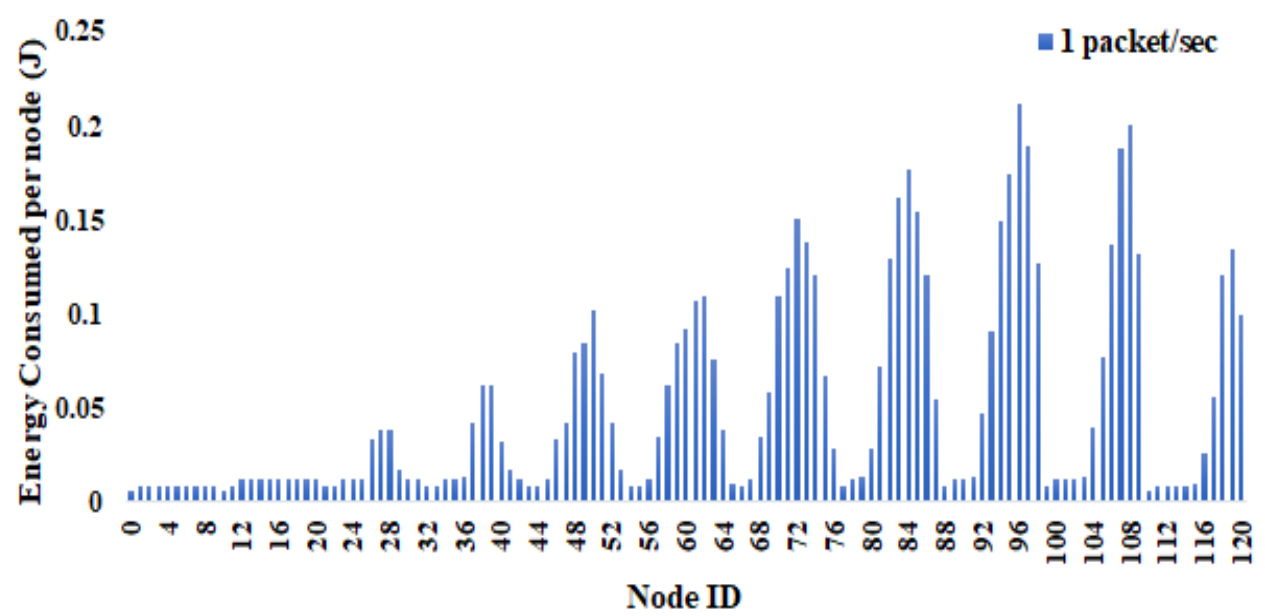

Figure.15 Static sink energy dissipation distribution in WSN (Joules) vs node ID for fixed packet rate

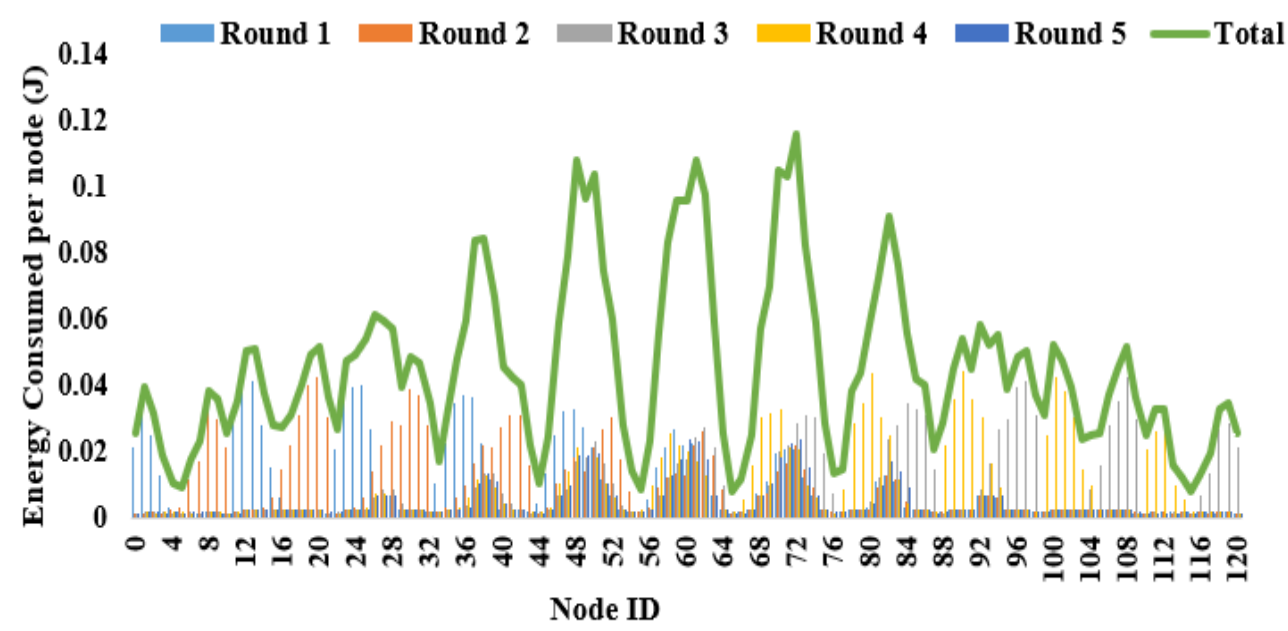

Figure.16 Quasi mobile sink energy dissipation distribution in WSN (Joules) vs node ID for fixed packet rate 


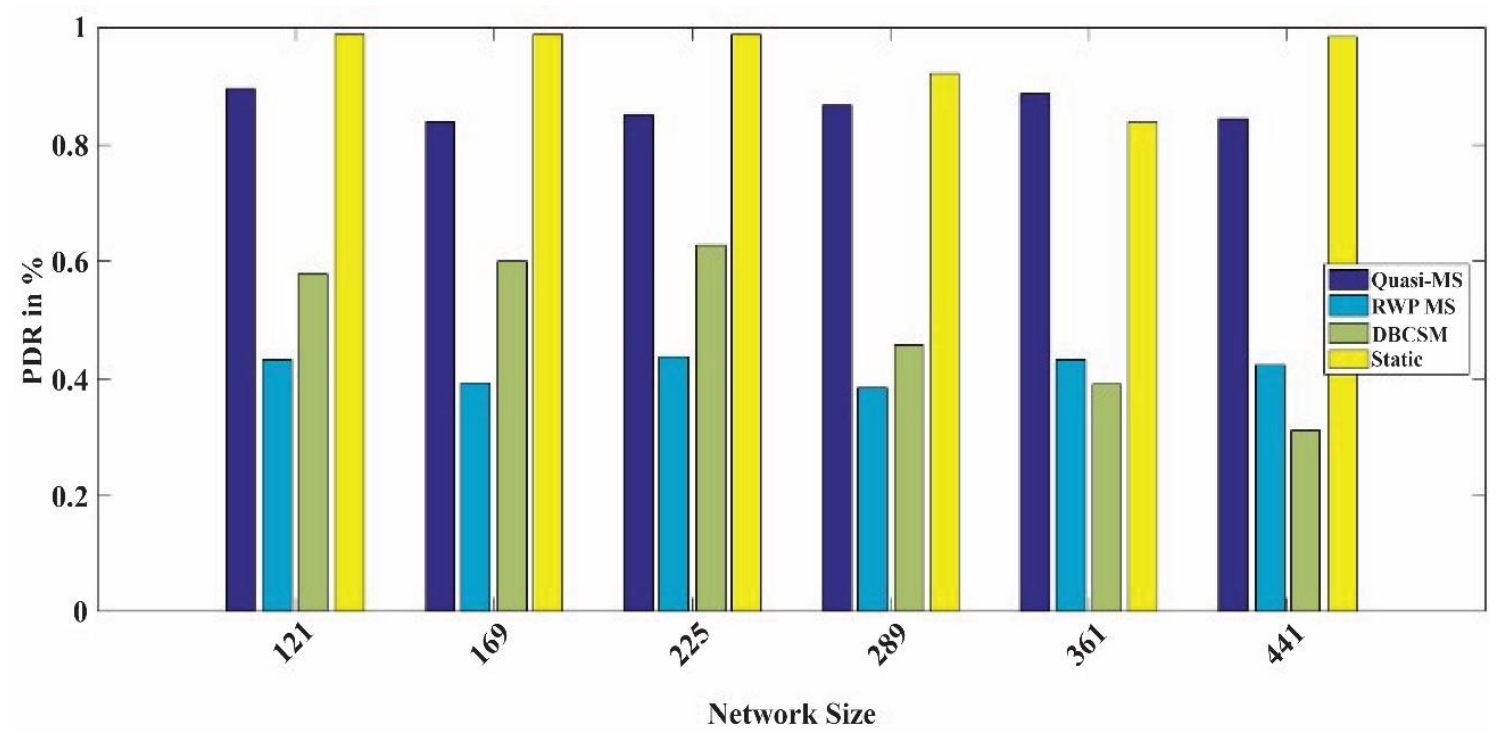

Figure.17 Packet delivery ratio (\%) vs network size

The average end to end delay is comparatively less around 2-4ms with respect to static sink for increase in packet rate up to 5 packets/second/node as shown in Fig. 14, due to the average reduction in the hop count achieved via Quasi-mobile sink model.

Fig. 16 shows that Energy dissipation distribution among the sensor nodes is nearly uniform in Quasi Mobile sink model and not heavily dissipated as seen in Fig. 15 (static sink solution).

\subsubsection{Simulation results and analysis with increase in network size}

The traffic rate with respect to query-matched source nodes was fixed and the network size was increased to evaluate the scalability of this model. The simulation results were compared with existing sink mobility models such as DBCSM [18], RWP [24] and static sink.

In the random way point (RWP) sink model [24], sink chooses random speed between minimum speed and maximum speed and moves toward a random destination to sojourn for a fixed pause time. The simulation was run for 5 random trials for each network size and statistical mean of performance metrics were obtained.

Distance based Controlled Sink Mobility (DBCSM) [18] consists of two stages namely distance calculation and movement stage. In the first stage, the distance from sink to each node is calculated and sink is made to move to the node's position that was not chosen by previous chosen node and far away by a distance (D), whose value depends on the number of nodes in the network.
However, in DBCSM, the sink keeps moving in controller manner to disseminate and collect the data. The conventional static sink solution, is where sink is stationary and fixed throughout the simulation.

Fig. 17 shows that Quasi MS (Mobile Sink) based approach has high packet delivery ratio compared to that of DBCSM and Random Waypoint which suffers badly due to link instability. The sojourn time for query dissemination and data gathering provides network stability and RSSI based parent selection with better link quality guarantees higher packet delivery ratio.

It is seen that Static Sink also achieves high packet delivery ratio due to same routing mechanism incorporated.

Fig. 18 shows that Average End to End delay scales up with increase in network size for Quasi-MS model but comparatively lesser than traditional static sink solution, since predefined sink movement patterns ensures network coverage and average reduction in hop count. Though RWP and DBCSM shows lesser delay and energy consumed (retransmissions not considered), it fails miserably in data delivery efficiency.

Fig. 19 infers that the average energy consumed in the network scales down with increase in network size for Quasi-MS model and it was shown in the previous section, that the energy dissipation is uniform in this model to avoid formation of hotspot unlike in static sink model 


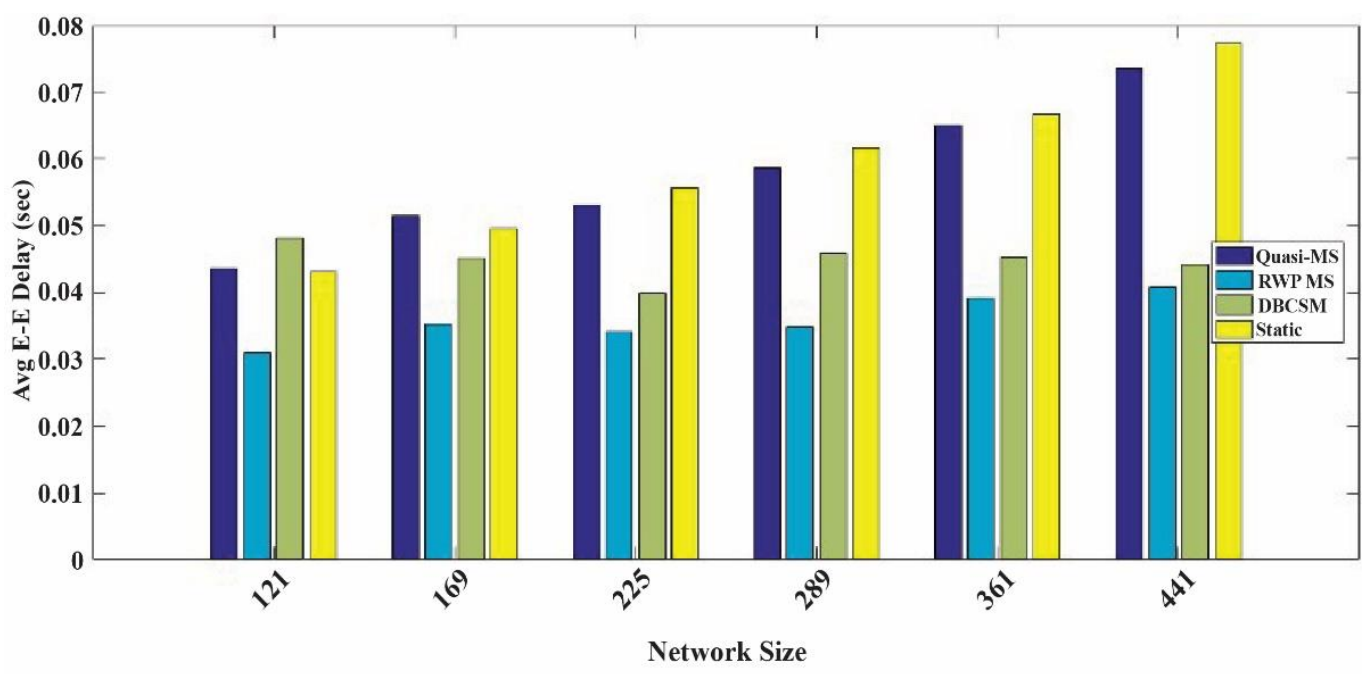

Figure. 18 Average end to end delay (s) vs network size

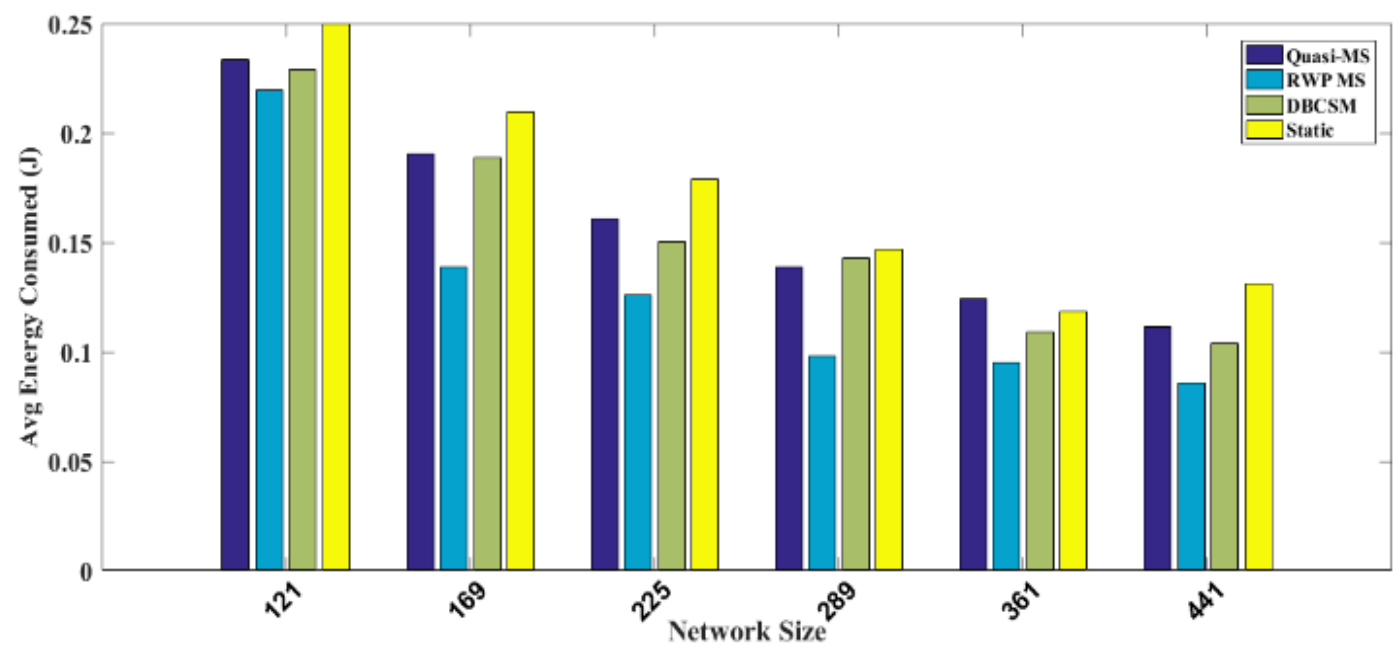

Figure. 19 Average energy consumed (J) vs network size

\section{Conclusion and future work}

This work proposed a novel Quasi Mobile Sink model to mitigate the hot-spot problem in large scale WSN. The limited number of sojourn rounds in this model is to balance the topological query update overhead and energy consumption in the network. A corona based flexible tree routing mechanism and RSSI based parent selection is adopted as routing metric in this work.

The simulation results show that Quasi Mobile sink based corona level routing mechanism scales well and achieves high packet delivery ratio compared with DBCSM and RWP based mobility models which suffers badly due to link instability. The average energy consumed in the network scales down with increase in network size and about 5-6.5\% increase in energy savings with increase in packet rate up to 5 packets/second per node is achieved among the hotspot region compared to traditional static sink model.

The energy dissipation distribution among sensor nodes is nearly uniform and not heavily dissipated compared to static sink model.

The average end to end delay is comparatively less around $2-4 \mathrm{~ms}$ with respect to static sink for increase in packet rate up to 5 packets/seconds/node due to average reduction in hop-count. Though DBCSM and RWP shows lesser end to end delay and energy consumed, it fails miserably in data delivery efficiency.

The optimal transmission power level selection algorithm was based on determining the maximum effective one-hop range with acceptable PSR such that the distance between sensor nodes is greater than the minimum separation distance to optimize overall deployment cost.

This work optimizes the deployment cost, energy cost and routing cost for scalable IoT driven periodic 
monitoring WSN applications. The future work includes adapting the quasi-mobile sink model for large scale mobile WSN applications under random deployment based on opportunistic multi-level tree routing mechanism.

\section{References}

[1] Y.G. Yue and P. He, "A comprehensive survey on the reliability of mobile wireless sensor networks: Taxonomy, challenges, and future directions", Information Fusion, Vol. 44, No. 11, pp. 188-204, 2018.

[2] X. Liu, Q. Huang, and Y. Zhang, "Balancing push and pull for efficient information discovery in large-scale sensor networks", IEEE Transactions on Mobile Computing, Vol. 6, No. 3, pp. 241-251, 2007.

[3] G. Khanna and S. K. Chaturvedi, "A Comprehensive Survey on Multi-hop Wireless Networks: Milestones, Changing Trends and Concomitant Challenges", Wireless Personal Communications, Vol. 101, No. 2, pp. 677-722, 2018.

[4] Z. Cheng, M. Perillo, and W. B. Heinzelman, "General network lifetime and cost models for evaluating sensor network deployment strategies", IEEE Transactions on Mobile Computing, Vol. 7, No. 4, pp. 484-497, 2008.

[5] A.U. Rahman, A. Alharby, H. Hasbullah, and K. Almuzaini, "Corona based deployment strategies in wireless sensor network: A survey", Journal of Network and Computer applications, Vol. 64, No. 4, pp. 176-193, 2016.

[6] I. Khoufi, P. Minet, A. Laouiti, and S. Mahfoudh, "Survey of deployment algorithms in wireless sensor networks: coverage and connectivity issues and challenges", International Journal of Autonomous and Adaptive Communications Systems, Vol. 10, No. 4, pp. 341-390, 2017.

[7] Y. Wang, Y. Zhang, J. Liu, and R. Bhandari, "Coverage, connectivity, and deployment in wireless sensor networks", in Recent Development in Wireless Sensor and Ad-hoc Networks, Springer: New Delhi, India, pp. 25-44, 2015.

[8] N. Ghosh and I. Banerjee, "An energy-efficient path determination strategy for mobile data collectors in wireless sensor network", Computers \& Electrical Engineering, Vol. 48, pp. 417-435, 2015.

[9] J. Luo and J.-P. Hubaux, "Joint sink mobility and routing to maximize the lifetime of wireless sensor networks: the case of constrained mobility", IEEE/ACM Transactions on Networking, Vol. 18, No. 3, pp. 871-884, 2010.

[10] S. Ghafoor, M. H. Rehmani, S. Cho, and S.-H. Park, "An efficient trajectory design for mobile sink in a wireless sensor network", Computers \& Electrical Engineering, Vol. 40, No. 7, pp. 2089-2100, 2014.

[11] A. W. Khan, A. H. Abdullah, M. H. Anisi, and J. I. Bangash, "A comprehensive study of data collection schemes using mobile sinks in wireless sensor networks", Sensors, Vol. 14, No. 2, pp. 2510-2548, 2014.

[12] R. Mitra and S. Sharma, "Proactive data routing using controlled mobility of a mobile sink in Wireless Sensor Networks", Computers \& Electrical Engineering, Vol. 70, pp. 21-36, 2018.

[13] A. Agrawal, V. Singh, S. Jain, and R. K. Gupta, "GCRP: Grid-cycle routing protocol for wireless sensor network with mobile sink", $A E U$ International Journal of Electronics and Communications, Vol. 94, pp. 1-11, 2018.

[14] S. Dahiya and P. K. Singh, "Optimized mobile sink based grid coverage-aware sensor deployment and link quality based routing in wireless sensor networks", AEU International Journal of Electronics and Communications, Vol. 89, pp. 191-196, 2018.

[15] A. A. Ahmed, "An enhanced real-time routing protocol with load distribution for mobile wireless sensor networks", Computer Networks, Vol. 57, No. 6, pp. 1459-1473, 2013.

[16] M. I. Khan, W. N. Gansterer, and G. Haring, "Static vs. mobile sink: The influence of basic parameters on energy efficiency in wireless sensor networks", Computer communications, Vol. 36, No. 9, pp. 965-978, 2013.

[17] L. Shi, B. Zhang, H. T. Mouftah, and J. Ma, "DDRP: an efficient data-driven routing protocol for wireless sensor networks with mobile sinks", International Journal of Communication Systems, Vol. 26, No. 10, pp. 1341-1355, 2013.

[18] M. A. Abdala and Z. A. Ismaeel, "Controlled Sink Mobility for Efficient Design of a Wireless Sensor Network", Journal of Telecommunications, Vol. 32, No. 2, pp. 1-4, 2016.

[19] K. Srinivasan, P. Dutta, A. Tavakoli, and P. Levis, "Understanding the causes of packet delivery success and failure in dense wireless sensor networks", In: Proc. of the 4th International Conference on Embedded Networked Sensor Systems, pp. 419-420, 2006.

[20] K. Levis and P. Levis, "RSSI is under appreciated", In: Proc. of the Third Workshop on 
Embedded Networked Sensors, Vol. 3031, pp. 239-242, 2006.

[21] O. Cheikhrouhou, G. M. Bhatti, and R. Alroobaea, "A Hybrid DV-Hop Algorithm Using RSSI for Localization in Large-Scale Wireless Sensor Networks", Sensors, Vol. 18, No. 5, pp. 1469, 2018.

[22] Digi International, "XBee/XBee-PRO S1 802.15.4", Data Sheet, 2018.

[23] "Low Power Radio Transceiver." www.ti.com/product/CC2420 (accessed on November 2018).

[24] T. Camp, J. Boleng, and V. Davies, "A survey of mobility models for ad hoc network research", Wireless communications and mobile computing, Vol. 2, No. 5, pp. 483-502, 2002. 